\title{
Magnetic drug delivery systems
}

\author{
Yang Liu, Mingxi Li, Fang Yang* and Ning Gu*
}

\begin{abstract}
There has been unprecedented progress in the development of biomedical nanotechnology and nanomaterials over the past few decades, and nanoparticle-based drug delivery systems (DDSs) have great potential for clinical applications. Among these, magnetic drug delivery systems (MDDSs) based on magnetic nanoparticles (MNPs) are attracting increasing attention owing to their favorable biocompatibility and excellent multifunctional loading capability. MDDSs primarily have a solid core of superparamagnetic maghemite $\left(\gamma-\mathrm{Fe}_{2} \mathrm{O}_{3}\right)$ or magnetite $\left(\mathrm{Fe}_{3} \mathrm{O}_{4}\right)$ nanoparticles ranging in size from 10 to $100 \mathrm{~nm}$. Their surface can be functionalized by organic and/or inorganic modification. Further conjugation with targeting ligands, drug loading, and MNP assembly can provide complex magnetic delivery systems with improved targeting efficacy and reduced toxicity. Owing to their sensitive response to external magnetic fields, MNPs and their assemblies have been developed as novel smart delivery systems. In this review, we first summarize the basic physicochemical and magnetic properties of desirable MDDSs that fulfill the requirements for specific clinical applications. Secondly, we discuss the surface modifications and functionalization issues that arise when designing elaborate MDDSs for future clinical uses. Finally, we highlight recent progress in the design and fabrication of MNPs, magnetic assemblies, and magnetic microbubbles and liposomes as MDDSs for cancer diagnosis and therapy. Recently, researchers have focused on enhanced targeting efficacy and theranostics by applying step-by-step sequential treatment, and by magnetically modulating dosing regimens, which are the current challenges for clinical applications.
\end{abstract}

Keywords: magnetic nanoparticles, magnetic assembly, drug delivery system, multimodality, theranostics

\section{INTRODUCTION}

Magnetic nanoparticles (MNPs), based on iron, cobalt, nickel, or metal oxides, have attracted significant attention owing to their intrinsic magnetic properties, which allow them to be tracked by magnetic resonance imaging (MRI) [1-4]. However, in the past few decades, the focus of laboratory researchers has shifted from pure material synthesis and characterization to the design of more comprehensive but practical therapeutic delivery systems. In recent years, integrated medical material for both diagnosis and simultaneous treatment has also become very attractive for doctors and patients because it can save a great deal of time and money. Based on this requirement, MNPs, previously used as a powerful diagnostic tool, are being considered as theranostic delivery systems combining imaging agents and effective therapeutic drugs [5-9].

Precise control parameters are pivotal in the synthesis and surface functionalization of MNPs because they determine the physicochemical properties, colloidal stability, and biological behavior and/or fate of the magnetic drug delivery systems (MDDSs). There is a large variety of MNPs, but superparamagnetic iron oxide nanoparticles (SPIO NPs) such as magnetite $\left(\mathrm{Fe}_{3} \mathrm{O}_{4}\right)$ and maghemite $\left(\gamma-\mathrm{Fe}_{2} \mathrm{O}_{3}\right)$ have great potential in nanomedicine. SPIO NPs have been widely utilized owing to their biocompatibility and "superparamagnetism". When exposed to an external magnetic field, they immediately reach saturation magnetization. When the external magnetic field is removed, they are demagnetized and do not retain any magnetism. SPIO NPs can be precisely directed to targeted tissue in vivo by exploiting their rapid response to external magnetic fields [10-15]. For specific pharmaceutical and biomedical purposes, MNPs should be modified by the incorporation of appropriate molecules on their surfaces. Owing to the large surface area of nanomaterials and their activated functional surface groups, it is very convenient to anchor a targeting agent to the MNP surface. Moreover, the number of targeting molecules can be readily controlled. For

State Key Laboratory of Bioelectronics, Jiangsu Key Laboratory for Biomaterials and Devices, School of Biological Science and Medical Engineering, Southeast University, Nanjing 210096, China

*Corresponding authors (emails: yangfang2080@seu.edu.cn (Yang F); guning@seu.edu.cn (Gu N)) 
example, biocompatible polymers such as polyethylene glycol (PEG) can be linked to the surface of MNPs to provide "stealth" properties while avoiding clearance by the reticuloendothelial system (RES). Target proteins such as herceptin can be conjugated on the surface of MNPs to facilitate their active delivery to breast tumors [15-18]. The design and fabrication of elaborate and advanced MNP-based DDSs is a promising approach to controlled loading and accurate multistep delivery [19].

MNPs are currently used in nanomedicine in different ways. For example, cancer chemotherapy requires the administration of high doses of cytotoxic drugs owing to their lack of specificity, which may lead to severe cytotoxic effects [20]. To avoid such side effects, MDDSs could be designed as smart drug delivery nanosystems to transport an effective drug dosage and specifically target tumor cells. The in vivo behavior of the drug could be further controlled by localizing it at the lesion site using an external magnetic field. Followed the application of another appropriate stimulation, the loaded drugs/imaging agents can be released locally [21-24]. In this review, we will summarize recent progress in the design and fabrication of multifunctional MDDSs for biomedical applications. After a brief introduction to the basic physicochemical and magnetic properties that are desirable for MDDSs, we will discuss the surface modification and functionalization issues that arise when designing elaborate MDDSs for future clinical applications. Finally, we will survey recent progress in the design and fabrication of controllable MNPs, magnetic assemblies, and magnetic microbubbles and liposomes for use in multimodal imaging and targeted drug delivery.

\section{PHYSICOCHEMICAL PROPERTIES OF MAGNETIC NANOPARTICLES FOR DRUG DELIVERY SYSTEMS}

To better serve in vivo applications, researchers have designed a series of MNPs with different combinations of cores and surface modifications. Firstly, it is necessary to have an overall understanding of how the physicochemical characteristics of MNPs affect their stability, pharmacokinetics, biodistribution, endocytotic pathway, and biotoxicity. By characterizing how geometry, hydrodynamic size, surface features, magnetism, and biotoxicity influence the delivery process (Fig. 1), researchers are able to redesign MDDSs with optimum therapeutic effects.

\section{Hydrodynamic size and geometry}

To achieve optimum efficacy, therapeutic agents must reach sufficient doses to kill tumor cells, but at the same time,

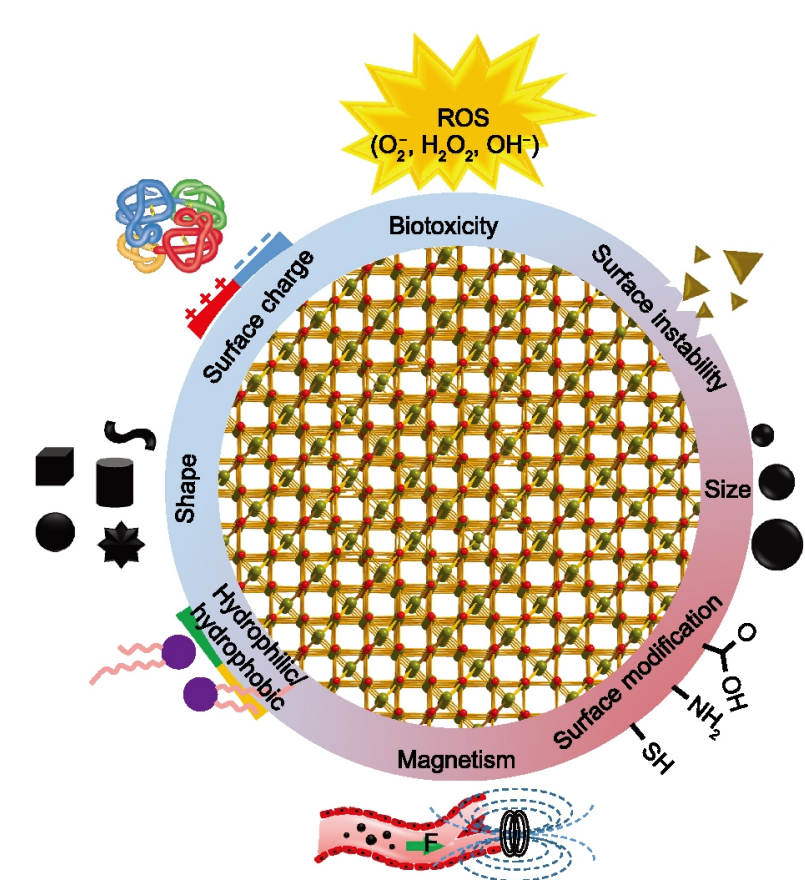

Figure 1 Physicochemical considerations of MNPs for drug delivery systems.

they should not adversely affect normal tissues. MNPs with large volumes or surface areas could carry sufficient drug molecules while avoiding clearance by RES. Therefore, shape and size must be carefully considered and optimized because they affect drug release and pharmacokinetics in vivo [25-27].

Circulation in the blood and extravasation of nanoparticles require the design of rational complex nanostructures in vivo. Small nanoparticles $(<5 \mathrm{~nm})$ might be rapidly removed from vessels by renal clearance or liver uptake, whereas large nanoparticles $(>200 \mathrm{~nm})$ are removed by the spleen or the RES $[28,29]$. Therefore, nanoparticles between 20 and $200 \mathrm{~nm}$ can be kept in blood circulation because they can escape from the body's scavengers [30]. Longer blood circulation time and higher plasma concentration ensure that nanoparticles can penetrate the epithelial cells in the vicinity of tumors and accumulate at a higher concentration at the targets. Furthermore, MNP drug delivery vehicles for theranostics, combined imaging, and hyperthermia treatment are also dependent on the magnetic properties of MNPs. Thus, the size of the MNPs should be carefully considered when designing MDDSs for in vivo applications.

Apart from the influence of MNP size, studies have shown that the shape of the nanoparticles may dictate the cellular fate of internalized MNPs [25]. Although few 
studies have focused on non-spherical nanoparticles to date, research has proved the plausibility of this speculation. Inhibition of phagocytosis by controlling shape succeeds by minimizing regions of high length-normalized curvature on the particles. Champion et al. [31] presented the idea that particle geometry can be used to modulate the phagocytosis of drug delivery particles, and demonstrated a significant reduction in particle uptake that was solely due to particle geometry. Worm-shaped nanoparticles, in particular, meet this criterion and have the potential to significantly affect drug delivery by avoiding phagocytosis compared with traditional spherical particles. Cheng et al. [32] demonstrated that the difference between uptakes of spherical MNPs and rod-shaped MNPs by HeLa cells might also be attributed to their morphological effects. Furthermore, MNPs with a high aspect ratio (from 100:1 to 500:1) have also been evaluated in vivo. The results revealed that constructs with a molecular weight of approximately $350-500 \mathrm{kDa}$ were rapidly (half life, $t_{1 / 2} \sim 6 \mathrm{~min}$ ) cleared intact by glomerular filtration; these constructs were much bigger than the molecular weight cutoff for glomerular filtration (30-50 kDa). Although the in vivo bio-behaviors of non-spherical MNPs may not be as predictable as we imagined, these observations have allowed the design of novel nanoscale-based shaped structures with unusual pharmacologic and pharmacokinetic characteristics [29].

\section{Surface features}

Once MNPs, which are distinct antigenic substances, are injected into the blood stream, and before they contact cells, they are recognized by the body's immune system. Their movement and release are affected by various cells and proteins in the blood. The MNP surface charge is regarded as one of the essential factors that directly relates to the cellular uptake of MNPs [23,33-35]. A surface charge is generated when most of the solid surface contacts water, an aqueous solution, or another highly polar liquid [36]. Owing to Coulombic force, the charged surface attracts solute ions of the opposite electric charge, resulting in the accumulation of high concentrations of counter ions at the interface. The Stern model postulates that a closely adsorbed ion layer, called the Stern layer, forms at the surface, and an outer layer, called the diffuse layer, also forms in which attraction decreases as distance increases. The potential of this double layer is called the zeta potential $(\zeta)$, and is a critical factor for the in vitro and in vivo stability of the MNPs. The zeta potential can be calculated indirectly by electrophoresis, photon correlation spectroscopy (PCS), and electroacoustic methods. In the macroscopic system, the effect of the surface charge between the solid-liquid interface is usually less significant and is neglected. However, under the micro-/nanosystem, the surface charge may have a critical effect on the behavior of the interface and the stability of colloidal particles. Understanding the structure of the electric double layer, and the effect of the surface charge on the interface of MNPs, will help us to more precisely control the surface charge to achieve the properties demanded by a specific application [37].

The surface charge of MNPs depends on the material coating them. For example, MNPs with a high number of amine groups are expected to have a positive charge, whereas hydroxyl and carboxyl groups usually confer a negative charge. Theoretically, because MNPs directly contact the charged head groups of proteins on the cell surfaces, positively charged MNPs are endocytosed by cells more easily, because the electronic potential of the cell membrane is negative. The experimental results produced by many researchers have proved this view. Yang et al. [38] determined that there was a greater concentration of positively charged (aminopropyltrimethoxysilane (APTMS)coated) MNPs inside cells than negatively charged (bare or tetraethyl orthosilicate (TEOS)-coated) MNPs. In particular, large numbers of APTMS-coated MNPs widely adhered to the immediate vicinity of the cell membrane, and several particles even translocated to the cell nucleus. Sun et al. [39] demonstrated that with a similar concentration, positively charged aminosilane-coated iron oxide nanoparticles (AmS-IONPs) reduced the viability of neurons by $50 \%$, whereas negatively charged $\mathrm{COOH}-\mathrm{AmS}$-SPIOs reduced viability by only $20 \%$. Toxicity appears to be dependent on the surface coating as opposed to the amount of iron oxide present in the cell. However, there is still some dissent among researchers regarding the zeta potential on the MNPs. Prijic et al. [40] proved that the cellular uptake of anionic silica-coated MNPs was three-fold greater than that of cationic-modified nanoparticles. Negative citrate groups increase the stability of MNPs and improve their affinity for the cell membrane. These contradictory experimental results illustrate the complex identification and phagocytosis process that occurs between cells and MNPs, in which surface charge is only one of many influencing factors.

\section{Magnetism}

As a DDS, MNPs should be capable of accumulating in selected regions to avoid adversely affecting the surrounding or non-targeted normal tissues. Therefore, MNPs are designed to have specific affinity for targeted regions through external guidance or internal stimuli. One of the most 
common ways of accomplishing this is to utilize the magnetic properties of the MNPs. Because the magnetic moments of the atoms of ferromagnetic materials are nonzero, each atom acts like a tiny permanent magnet. When the atoms are clustered into a small region, and the magnetic moments are evenly arranged in parallel, the small region is called the magnetic domain [41]. The existence of magnetic domains is a consequence of minimizing energy. Assuming that a ferromagnetic bulk comprises multiple magnetic domains, the magnetic fields of the magnetic domains are different and cancel each other out, resulting in a zero-sum vector. Because the magnetic moment of the whole object is zero, it cannot attract other magnetic materials. However, if the ferromagnetic material is exposed to an external magnetic field, the magnetic domain starts to move. If the direction of the magnetic domain is approximately the same as the direction of the external magnetic field, the magnetic domain expands; if the directions are different, it contracts. At this point, if the magnetic field is switched off, the magnetic domain may not return to the original unmagnetized state. Therefore, when the ferromagnets are small enough, the nanoparticles randomly change direction owing to thermal perturbation. When there is no external magnetic field, they usually do not exhibit magnetic properties. However, once an external magnetic field is applied, the MNPs become magnetized, a phenomenon known as superparamagnetism [42].

In the absence of an external magnetic field, superparamagnetic materials do not retain residual magnetism; this property could be exploited to avoid the tendency to agglomerate in colloidal dispersions [43]. In fact, researchers have demonstrated that reduced intrinsic ferromagnetism ensures non-toxicity under physiological conditions. Furthermore, driven by external magnetic fields, MDDSs can provide controllable movement, accumulation, and release within the organism [44]. For instance, some researchers have found that magnetic composites can be localized upon the application of an external magnetic field and can circumvent the human skin barrier, and even penetrate deep subcutaneous tissue via follicular pathways [20].

Moreover, by responding to an external alternating magnetic field, MNPs can convert dissipated magnetic energy into thermal energy. Thus, it is envisaged that MNPs will be used for hyperthermia treatment of cancer or hyperthermia-controlled drug release [13,45-47]. MNPs generate heat under an alternating magnetic field as a result of various pathways strongly correlated with their morphological, structural, and magnetic profiles. As shown in previous studies [48], magnetic hyperthermia efficiency may be enhanced by controlling the core-shell morphology of mixed ferrites compared with their single-phase counterparts. More thorough research is required in order to obtain better energy conversion efficacy.

\section{Biotoxicity}

When MNPs are engineered for use as biomedical DDSs, their toxicity has also been a concern. As prerequisites for clinical application, complete knowledge of the toxicological properties and a risk assessment of the MNPs are mandatory to ensure safety and minimize potential health hazards.

At present, MDDSs are commonly designed with a core of magnetic nanomaterials and a shell of polymer materials or other inorganic metal compounds. Methods for in vivo detection of MNPs are mainly based on the determination of iron content using elemental analysis of collected blood [49]. However, because most biomedical MNPs are iron-based [50], the quantitative determination of the iron content to obtain an understanding of the pharmacokinetics of the MNPs is complicated by the presence of endogenous iron. However, some new technologies such as alternating current magnetic susceptibility measurements facilitate differentiation between the endogenous iron present in the tissues in the form of ferritin and the iron storage protein from MNPs [51]. Ruiz et al. [51] demonstrated that meso-2,3-dimercaptosuccinic acid (DMSA)-coated iron oxide nanoparticles are safe for biomedical applications. They administered the nanoparticles to rats at dose levels of 2.5, 7.5, and $15 \mathrm{mg} \mathrm{Fe} / \mathrm{kg}$ body weight and monitored their body weight, food consumption, gross pathology, and the bio-distribution of iron in their spleens and livers. Many studies have compared the toxicity of different magnetic nanomaterials during cell coculture [52-56]. Park et al. [55] proved that after the cells were exposed to $50 \mu \mathrm{g} \mathrm{mL} \mathrm{L}^{-1}$ of bare magnetite and maghemite for $24 \mathrm{~h}, \mathrm{Fe}_{3} \mathrm{O}_{4}$ decreased cell viability to 11.0 $\pm 1.0 \%$ of the control level. Adenosine monophosphate (ATP) production decreased to $9.5 \pm 0.6 \%$ of the control level. The levels of reactive oxygen species, nitric oxide, and pro-inflammatory cytokines were elevated. Damage to the mitochondria and the endoplasmic reticulum, and downregulation of mitochondrial function and transcription-related genes were also higher in the cells. However, although $\gamma-\mathrm{Fe}_{2} \mathrm{O}_{3}$ produced the same results, the degree of the effect was lower.

Therefore, to reduce toxicity, the iron core is generally coated with biocompatible materials such as dextran, DMSA, PEG, or polystyrene [57-59], which will be de- 
scribed in detail in the next section. Researchers even hope to use biocompatible modifications to avoid unnecessary tissue aggregation and cell endocytosis, thereby ensuring the biosafety of the MDDS. For example, dextran-stabilized MNPs do not induce oxidative stress-mediated toxicological effects, nor do they alter physiological processes or behaviors, or visible pathological lesions [60]. Peeples et al. [61] showed that coating $\mathrm{Fe}_{3} \mathrm{O}_{4}$ nanoparticles with (3-aminopropyl) triethoxysilane and citric acid caused no significant decrease in cell viability up to $25 \mu \mathrm{g} \mathrm{mL}^{-1}$ compared with uncoated $\mathrm{Fe}_{3} \mathrm{O}_{4}$ when incubated with lung epithelial cells (RL 65-Rat source). The experimental analysis of the effects of DMSA-modified MNPs on higher concentrations and longer incubation times also showed that DMSA-MNPs have little toxicity in this cell line. There is no notable influence on cell proliferation. It was further proved that treatment with various concentrations of DMSA-MNPs led to an insignificant decrease in glutathione (GSH) levels [62]. These results confirm the potential of surface coated MNPs for clinical use.

In addition to directly designing iron nanoparticles as drug carriers, the magnetic responsive capability of MNPs is often used to affect drug release under external magnetic field stimulation. Therefore, a combination of MNPs with static magnetic field (SMF), or alternating magnetic field may cause potential negative effects for clinical applications. Unfortunately, there are very few reports on this problem. Bae et al. [63] reported that under conditions that satisfied the conventional cytotoxicity assessment of $0.5 \mathrm{mmol} \mathrm{L}^{-1} \mathrm{SPIO}$, clinical doses combined with $0.4 \mathrm{~T} \mathrm{SMF}$ exposure exert synergistic adverse effects such as reduced cell viability, apoptosis, and cell cycle aberrations on hepatocytes in vitro and in vivo. Moreover, long-term monitoring showed a significant increase in multinuclear giant cells in the cells concomitantly treated with SPIO and SMF compared with the control. Therefore, further studies on the molecular mechanisms underlying cellular responses to both MNPs and magnetic field interactions are necessary for a comprehensive assessment and a thorough understanding of the systemic effects on the living body.

\section{SURFACE FUNCTIONALIZATION OF MAGNETIC NANOPARTICLES FOR IN VIVO TARGETING}

To improve stability, reduce biotoxicity, and achieve extended circulation under physiological conditions, MNPs need to be functionalized by encapsulating a variety of inorganic or organic materials such as polymers, lipids, and proteins, or further conjugating functional moieties to their coatings such as targeting ligands, therapeutic peptides/antibodies, fluorescent dyes, or gene agents. Table 1 summarizes common organic and inorganic surface modifications for better in vivo results. The surface modifications can provide protection for the magnetic core, preventing aggregation by screening the magnetic dipolar attraction between the MNPs. They ensure an ideal magnetic platform for further drug loading and retain chemical inertness with normal tissues in biological systems.

\section{Organic coating materials}

\section{Polymer coating materials}

Improving the targeting efficiency of MNPs while reducing their negative impact in vivo has always been a challenge for MDDS applications. To solve this problem, MNPs for in vivo use require a coating that: (1) reduces MNP aggregation; (2) extends the circulation time of the MNPs in vivo; (3) avoids nonspecific intercellular interactions and reduces cytotoxicity; and (4) provides a platform for the conjugation of drugs and targeting molecules. To achieve these coating functions, various polymers including PEG, poly(ethyleneimine) (PEI), chitosan, polylactic acid (PLA), and dextran have been investigated.

PEG has several advantages, such as low toxicity, no antigenicity, good amphiphilicity and biocompatibility, and thus has been used clinically as an excipient in Food and Drug Administration (FDA)-approved pharmaceutical formulations [74]. PEG-coated (or PEGylated) MNPs can usually avoid recognition by the RES, thereby improving the biocompatibility of MNPs. PEG can be further combined with certain proteins, targeting ligands, or therapeutic agents. Even by covalently binding to the other end of PEG, such magnetic complexes can achieve both efficient surface coating and multi-functionalization. Yuan et al. [75] prepared well-defined PEGylated MNPs $\left(\mathrm{PEG}-\mathrm{Fe}_{3} \mathrm{O}_{4}\right)$ with excellent dispersibility and dissolvability under physiological conditions for the photothermal therapy of cancer cells via a facile one-pot solvothermal method. Various hybrid MNPs coated with PEG or carboxylated PEG [76], such as PEGylated iron oxide-gold core-shell nanoparticles [77], combine a variety of specific ligands for applications in diagnostic magnetic particle imaging (MPI) [78], MRI [79], and the treatment of many diseases.

Chitosan, which is a partially acetylated glucosamine (poly(1 $\rightarrow 4)$-2-amino-2-deoxy-d-glucan) [80], was firstly synthesized in 1859 . This natural polymer has bio-functionality, biodegradability, biocompatibility, bioactivity, and other excellent properties, and has attracted attention 
Table 1 Materials used to modify the surface of MNPs for in vivo applications

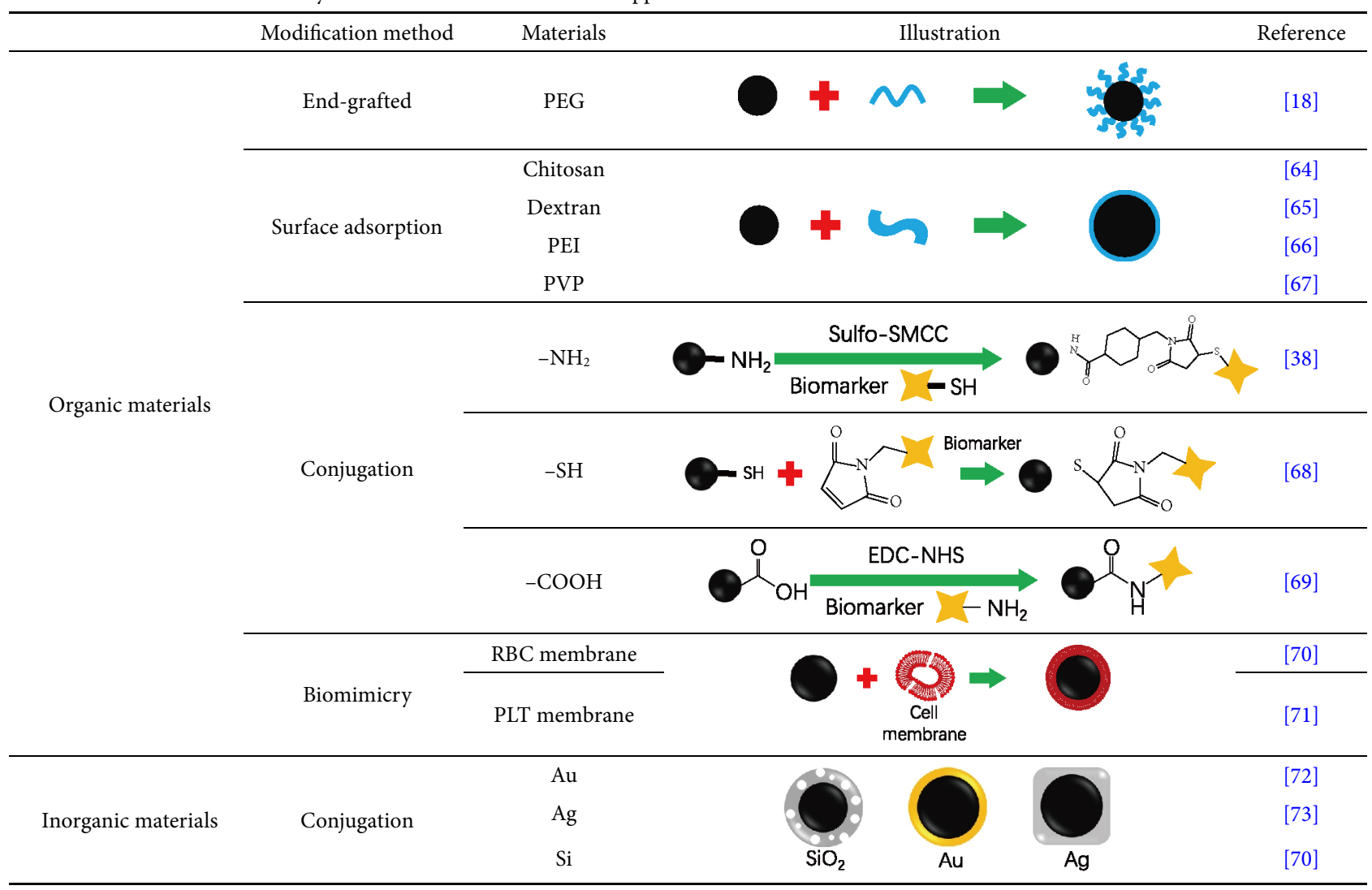

Note: $\mathrm{PEG}=$ polyethylene glycol; $\mathrm{PEI}=$ poly $($ ethyleneimine $) ; \mathrm{PVP}=$ polyvinylpyrrolidone; $\mathrm{RBC}=$ red blood cell; $\mathrm{PLT}=$ platelets

in the fields of chemistry and biology. Chitosan-coated MNPs contain a core of magnetic material, usually a mixture of $\mathrm{Fe}_{3} \mathrm{O}_{4}$ and $\gamma-\mathrm{Fe}_{2} \mathrm{O}_{3}$ [81], or hybrid MNPs (nickel-ferrite) [82], with the drug loaded on the surface for targeted, controlled release. Dorniani et al. [83] designed a new drug nanocarrier by coating chitosan and perindopril erbumine on the surface of MNPs using a simple coating method. Such chitosan-modified MDDSs have minimal toxicity. In addition, chitosan has reactive groups such as $-\mathrm{OH}$ and $-\mathrm{NH}_{2}$, which can be used for further integration of MNPs with targeting, imaging, and therapeutic agents.

PEI is a hydrophilic cationic polymer with high affinity for DNA, proteins, and cells [84]. It is often used in conjunction with PEG for coating MNPs to enhance colloidal stability and reduce biotoxicity. Schweiger et al. [85] developed a novel magnetic carrier system based on the assembly of PEG-PEI and MNPs to develop $\gamma-\mathrm{Fe}_{2} \mathrm{O}_{3}$ PEG-PEI particle systems. Such nanodelivery systems have good long-term colloidal stability and low toxicity in the presence of PEG groups on the polymer backbone, and have great potential for biomedical applications.
Dextran is a water-soluble polysaccharide that is mainly composed of $a$-d-( $(1 \rightarrow 6)$-linked glucose units with some $\alpha$-d-(1 $\rightarrow 3)$-linked units [57]. As a natural polysaccharide, dextran is widely used in the pharmaceutical field and has attracted much attention [86]. It has a strong affinity for the hydroxyl groups on the surface of MNPs. Dextran-modified MNPs can increase the half-life of the drug under physiological conditions. Many dextran-coated MNPs have been prepared for use as MRI contrast agents and targeted drug delivery carriers because they have good biocompatibility $[58,59,87,88]$. Dextran can also be coated on hybrid MNPs. The dextran-coated gold MNPs synthesized by Li et al. [89] had enhanced colloidal stability and could be used for the controlled release of doxorubicin (DOX).

A variety of other polymers or copolymers are also used for the encapsulation of MNPs, including polyvinyl alcohol (PVA) [90,91], polylactic acid/poly(lactic-co-glycolic acid) (PLA/PLGA) [92], and polyvinylpyrrolidone (PVP) [93-96]. These polymers have different properties and can influence the properties of MNPs, such as the surface 
charge, function, dispersibility, and magnetic properties, in different ways. Modified MNPs can successfully endure the aggregation of particles under physiological conditions and retain a good crystalline structure and magnetic properties, which make them ideal for controlled drug delivery in tumor therapy.

\section{Bioconjugation coating materials}

Coating strategies using polymers such as PEG and PLA to improve the surface properties of MNPs have been highly successful at solving the biocompatibility problem. The further bioconjugation of functional structures on the surface of MNPs has been reported to enhance targeting capability. Wang et al. [97] designed (3-aminopropyl)triethoxysilane-modified FeCo MNPs, which were subsequently activated by glutaraldehyde, leading to the successful bioconjugation of proteins (streptavidin, pregnancy-associated plasma protein A antibody, and nectin-4 antibody) with the aldehyde groups on the nanoparticle surfaces. Protein-FeCo conjugates have much higher saturation magnetization than commercially available iron oxide nanoparticles. In addition, some protein drugs/genes can also be used to modify the surface of MNPs to achieve the targeted delivery of biological drugs. Yang et al. [98] developed low-toxicity magnetic nanocarriers with a shell of poly(aniline-co- $\mathrm{N}$-(1-one-butyric acid) aniline) over a $\mathrm{Fe}_{3} \mathrm{O}_{4} \mathrm{MNP}$ core to carry recombinant tissue plasminogen activator (rtPA) for targeted thrombolysis treatment.

\section{Natural biological coating materials}

The modulation of immunocompatibility by the chemical modifications described above is limited owing to exogenous toxicity. Recent studies on the immunological response of artificial material-modified nanoparticles have prompted researchers to pursue alternatives. A cell membrane-based top-down nanoparticle modification strategy has proved successful. Rao et al. [99] made use of natural red blood cell (RBC) membranes to camouflage the surface of $\mathrm{Fe}_{3} \mathrm{O}_{4}$ nanoparticles for reducing RES uptake. The combination of MNPs and natural cell membranes embodies a biomimetic nanocoating strategy for designing new biological magnetic nanomaterials. Owing to their self-recognition function, autologous cells that mimic the MDDS strategy have a great advantage in biomedical applications. Mimicking MNPs with other types of autologous cell membranes such as those of leukocytes, platelets, cancer cells, hepatocytes, and stem cells is a current research hot spot.

\section{Inorganic coating materials}

Owing to the combination of the magnetic core and the functionalized shell, hybrid MNPs with a core-shell structure have a wide range of applications $[100,101]$. Many inorganic materials such as $\mathrm{SiO}_{2}$, or precious metals such as gold, silver, or platinum, are used for the nuclei or shells of MNPs. These coated hybrid MNPs have enhanced stability, improved biocompatibility, and surface chemical, biological, or catalytic interfacial reactivity.

\section{Silica-coated MNPs}

Recently, core-shell structured silica/MNP composites have been studied extensively [102-104]. Silica-coated MNPs can be used for MRI imaging and hyperthermia treatment. The preparation of silica-coated MNPs with good physical and chemical properties is a prerequisite for subsequent use. Rho et al. [105] described a facile two-step method for synthesizing monodispersed, silica-coated MNPs. Oleate-MNPs were successfully converted to polyvinylpyrrolidone-MNPs (PVP-MNPs), which were then coated with silica using a modified version of the Stöber method. More than $95 \%$ of the MNPs were individually coated with a silica shell without non-magnetic core silica nanoparticles, which were stable for more than three months. Studies on silica-coated MNPs indicate that these particles have the potential for use in biomedical applications. Owing to the presence of MNP cores, silica-coated MNPs can be used for hyperthermia treatment. Iqbal et al. [102] prepared silica-coated manganese ferrite nanoparticles for hyperthermia applications. Silica-coated manganese ferrite nanoparticles can be used to heat aqueous solutions to $42^{\circ} \mathrm{C}$, and are therefore useful for magnetic hyperthermia treatment. Compared with research into organic coating materials such as PEG, PEI, and lipids, there has been little research into silica as an inorganic coating material for MNPs until recently. Silica-coated MNPs may be useful in various biomedical fields such as diagnostics and therapeutic treatments because they are biocompatible, stable, non-toxic, easily functionalized, and have excellent magnetic properties.

\section{Gold-coated MNPs}

Gold $(\mathrm{Au})$-coated MNPs with a core-shell structure can be used for MRI, magnetically targeted drug delivery, surface-enhanced Raman scattering (SERS), and catalysis. Owing to their unique properties, gold-coated magnetic composites can be used for both diagnosis and therapeutics. Shen et al. [106] recently reported the design of gold-coated $\mathrm{Fe}_{3} \mathrm{O}_{4}$ nanoparticles. The nanoparticles were functionalized by the self-assembly of a single layer of azide groups on the surface, which could be conjugated with folate molecules via copper (I)-catalyzed azide-alkyne 
cycloaddition. The experimental results showed that the nanoparticles could be used for the non-immunogenic targeting of cancer cells. Owing to the multifunctional modification of Au-coated MNPs, multimodal imaging and photothermal therapy can be envisaged in the future. In addition to $\mathrm{Fe}_{3} \mathrm{O}_{4}$, the core of gold-coated MNPs can be synthesized from other hybrid materials. Poudyal et al. [107] reported a novel method for preparing a FePtAu core-shell structure using solution phase chemistry combined with solvothermal annealing, which showed great promise for various optical, sensing, and biomedical applications. Wang et al. [108] designed Au-coated $\mathrm{MnFe}_{2} \mathrm{O}_{4}$ nanoparticles, which were first modified with a uniform PEI layer $(2 \mathrm{~nm})$. The negatively charged $\mathrm{Au}$ seeds were then adsorbed onto the surface of the $\mathrm{MnFe}_{2} \mathrm{O}_{4}$ nanoparticles via electrostatic interaction during the formation of the Au shell. Synthetic Au-coated hybrid MNPs have useful properties such as strong magnetic response, good SERS activity, enhanced stability, and biofunctionalization. Moreover, the thickness of the gold layer can be varied as required to achieve different properties, making the nanoparticles potentially useful for a broad range of applications in diagnosis and therapeutics.

\section{Silver-coated MNPs}

When silver (Ag) is combined with MNPs, silver-MNP composites with both optical and magnetic properties are obtained. The biocompatibility of the nanoparticles can be improved. Chen et al. [109] reported the one-pot synthesis of $\mathrm{Ag}-\mathrm{Fe}_{2} \mathrm{O}_{3}$ hybrid nanoparticles by the sequential addition of precursor chemicals. It is possible to adjust the hybrid structure from a core-shell to a heteromeric geometry by changing the reaction temperature. Owing to the slow diffusion of silver ions out of the $\mathrm{Fe}_{2} \mathrm{O}_{3}$ shell, the hybrid material has an enhanced magnetic-targeting, bactericidal function. Zhai et al. [27] were the first to describe a solvothermal method whereby $\mathrm{Fe}_{3} \mathrm{O}_{4}$ grains were distributed directly onto the surface of $\mathrm{Ag}$ seeds. They constructed $\mathrm{Ag}-\mathrm{Fe}_{3} \mathrm{O}_{4}$ hybrid MNPs with a core-shell structure, which had both plasmonic and significant superparamagnetic properties. Silver-iron hybrid MNPs can also be synthesized by the in situ reaction of precursor chemicals. Bian et al. [110] prepared monodispersed $\mathrm{Ag} /$ polyaniline $/ \mathrm{Fe}_{3} \mathrm{O}_{4}$ nanoparticles with an average size of approximately $50 \mathrm{~nm}$ via in situ reduction between emeraldine $\mathrm{PANI} / \mathrm{Fe}_{3} \mathrm{O}_{4}$ and silver nitrate. Magnetite-silver hybrid nanoparticles can easily be tuned to a core-shell or heteromer structure [25], and show great potential for application in the fields of tumor treatment using magnetic hyperthermia, SERS, antibacterial applications, and optical imaging.

\section{MAGNETIC NANOPARTICLES AND ASSEMBLED COMPOSITES AS DRUG DELIVERY SYSTEMS FOR THERANOSTICS}

The inability of traditional drug delivery carriers to specifically accumulate at the target site and escape the biological barriers reduces drug efficiency and stability, and can even lead to serious side-effects. Therefore, the use of biocompatible carriers to carry therapeutic drugs with improved pharmacokinetic properties has attracted the attention of researchers. MNP-assembled MDDSs have been regarded as an attractive alternative for delivering drugs owing to their low toxicity, biocompatibility, and controllable release characteristics. Although it is well known that MNPs can be used as MRI contrast agents for tumor imaging, recent multifunctional MDDSs have extended the potential uses of MNPs by combining multimodal imaging with targeted drug delivery; the MNPs can be loaded with radiotherapy, chemotherapy, anti-inflammatory, or anticancer drugs.

Magnetic nanoparticles themselves as a drug delivery system In the early stages of MNP development, researchers regarded MNPs as chemically inert materials, and they have mainly been used as MRI contrast-enhancing agents and spontaneous DDSs. However, subsequent studies have revealed that MNPs have $\mathrm{pH}$-dependent peroxidase and catalase activities like those of certain enzymes. Chen et al. $[111,112]$ reported that iron oxide nanoparticles can catalyze $\mathrm{H}_{2} \mathrm{O}_{2}$ to produce the hydroxyl radical $(\cdot \mathrm{OH})$ under acidic conditions, and the hydroxyl radical is then able to oxidize a variety of organic molecules. That is, the MNPs have peroxidase-like activity. Under neutral conditions, iron oxide nanoparticles directly catalyze the degradation of hydrogen peroxide into $\mathrm{H}_{2} \mathrm{O}$ and $\mathrm{O}_{2}$ (Fig. 2); that is, they have hydrogen peroxidase-like enzyme activity. Such $\mathrm{pH}$-dependent enzyme activity of MNPs has prompted new lines of investigation into the use of MNPs themselves as "drugs" for the treatment of disease.

An attempt has been made to exploit the enzymatic activities of MNPs to treat certain diseases. Xiong et al. [113] reported the preparation of the DMSA-coated $\mathrm{Fe}_{2} \mathrm{O}_{3}$ MNPs ( $\left.\mathrm{Fe}_{2} \mathrm{O}_{3} @ D M S A\right)$, which have a spherical core with an average diameter of $9.8 \mathrm{~nm}$, using a coprecipitation method. Importantly, $\mathrm{Fe}_{2} \mathrm{O}_{3} @ D M S A$ showed the potential for drug-like activity, preventing cardiac arrest in a rat coronary artery ligature model. The size of the myocardial infarction and the biochemical indices further demon- 


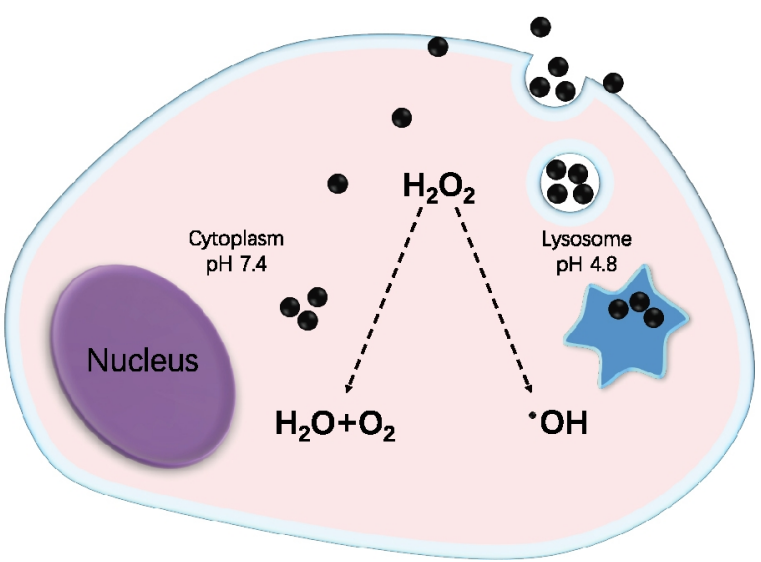

Figure 2 Schematic illustration of peroxidase- and hydrogen peroxidaselike enzyme activities of iron oxide nanoparticles, modified from reference [111], Copyright 2012, American Chemical Society.

strated that $\mathrm{Fe}_{2} \mathrm{O}_{3} @ D M S A$ nanoparticles can successfully protect the myocardium from ischemia injury in animals. Further, it is found that the MIM, a multifunctional scaffold protein to regulate both actin dynamics and membrane dynamics, may play a positive role in the MNPs uptake process. Therefore, the silence of MIM is important for avoiding the endocytosis process of MNPs by RAW 264.7, which may enhance the MNPs accumulation in target sites [114].

The discovery of the mimetic enzyme activity of MNPs has prompted researchers to explore new strategies for their synthesis, laying the foundation for further MNP applications. There have been some reports that naturally occurring ferritin is equivalent to a storage compartment for iron in animal and plant cells [115]. Zhang et al. [116] devised a strategy to use ferritin as a template for the synthesis of small, Prussian blue-modified ferritin nanoparticles (PB-Ft NPs), which retain the biological characteristics of ferritin. Their results showed that the PB-Ft NPs have a mean size of $22.8 \mathrm{~nm}$, and retain specificity and peroxidase-like activity. Therefore, PB-Ft NPs can be used as biocatalytic and biometric tools. However, contrary to widespread speculation, the electron spin resonance test results show that PB-Ft NPs cannot catalyze $\mathrm{H}_{2} \mathrm{O}_{2}$ to produce $\cdot \mathrm{OH}$. On the contrary, PB-Ft NPs clean up harmful $\cdot \mathrm{OH}$, which indicates that PB-Ft NPs do not act as peroxidases through the Fenton reaction. Further experimental results reported by Zhang et al. [117] show that PB-Ft NPs exert a catalytic function through a charge transfer mechanism. Moreover, PB-Ft NPs can effectively quench $\mathrm{O}_{2}$ and $\mathrm{H}_{2} \mathrm{O}_{2}$ in several cell models, i.e., PB-Ft NPs act as nanoscale reactive oxygen species (ROS) scavengers that effectively relieve ROS-induced cell damage. However, the detailed cellular mechanism underlying this property of PB-Ft NPs is unclear and requires further investigation. Moreover, Yang et al. [118] have developed a new strategy of ultrasound and MR dual modal imaging, which has a complementary advantage that enables simultaneous treatment and multimodal monitoring based on PB-Ft NPs. PB-Ft NPs are able to catalyze the decomposition of $\mathrm{H}_{2} \mathrm{O}_{2}$ into oxygen $\left(\mathrm{O}_{2}\right)$ molecules at neutral $\mathrm{pH}$ (pH 7.4). This chemical reaction produces an $\mathrm{O}_{2}$ bubble-forming molecule that can be used as an ultrasound contrast agent to enhance ultrasound imaging. Thus, PBNPs provide a multi-functional nanoscale platform; they can be used for dual-mode imaging and as scavengers to reduce oxidative stress in vivo.

The results mentioned above suggest that the biological activity of MNPs may be very complex. Some researchers have reported that MNPs have enzymatic activity that resembles the effects of certain drugs, and can also promote the osteogenic differentiation of human bone marrow mesenchymal stem cells (hBMSCs) to modulate stem cell fate for promoting tissue repair [119,120]. Wang et al. [121] analyzed the gene expression of hBMSCs that had been incubated with MNPs at an appropriate concentration using a gene chip assay and bioinformatics analysis. They found that the classical mitogen-activated protein kinase (MAPK) signaling pathway was activated by NPs. Thus, the downstream gene of the pathway is regulated to promote osteogenic differentiation. This study elucidates the molecular basis of how MNPs affect hBMSCs, which may have significant implications for stem cell applications in regenerative medicine.

\section{Magnetic nanoparticle assembly as a drug delivery system}

Employing MNPs as a DDS is another common use of MNPs. MNPs are able to transport anticancer drugs into tumor cells without damaging healthy cells. Compared with other nanodrug delivery systems such as polymer nanoparticles, liposomes, and micelles, MDDSs have better MRI performance, which may make it possible to dynamically monitor the drug distribution in vivo. Furthermore, MNPs are biodegradable, which greatly benefits their biomedical applicability. However, the efficacy of the MNPs as drug nanocarriers is often counteracted by the rapid conditioning and subsequent plasma clearance of the tissue macrophages of the RES before the nanoparticles reach the target tissue or cell [122]. Although we have developed many ways of loading MNPs with drugs, magnetic carriers may perform poorly when a large drug release 
at the target area is required. Smaller particles provide a larger surface area and are therefore more suitable for drug loading, but this comes at the expense of reduced magnetic properties. To solve this problem, Xiong et al. [123] assembled four separate oleic acid-terminated iron oxide nanotubes and oleic acid-modified PEG molecules to form clusters (Fig. 3). These nanomagnetic clusters maintained high paclitaxel (PTX) drug loading, high magnetism, and rapid and extended release behavior. Compared with the same dose of free PTX, the PTX magnetic nanoassemblies had greater antitumor activity in vivo. Simultaneously, with the increase of tumor cell uptake, the magnetic nanoassemblies provided tumor imaging by MRI. Benefiting from the high drug loading capacity and high magnetic characteristics, such magnetic nanoassembled DDSs can maximize the advantages of nanomaterials and minimize their side-effects.

Moreover, biodegradable and biocompatible polymerassembled MNP structures have been fabricated based on their controlled drug release characteristics, and have been used in biomedical imaging, cell labeling, and therapy [124]. Yang et al. [125] found that MNP-embedded PLA exhibited controlled drug release that was dependent on the MNP assembly concentration in the polymer. PLA-MNP material with a concentration of $20 \%$ MNPs in the composite increased the drug release rate by more than 200 times while maintaining excellent controlled drug release. It was also found that the interaction between

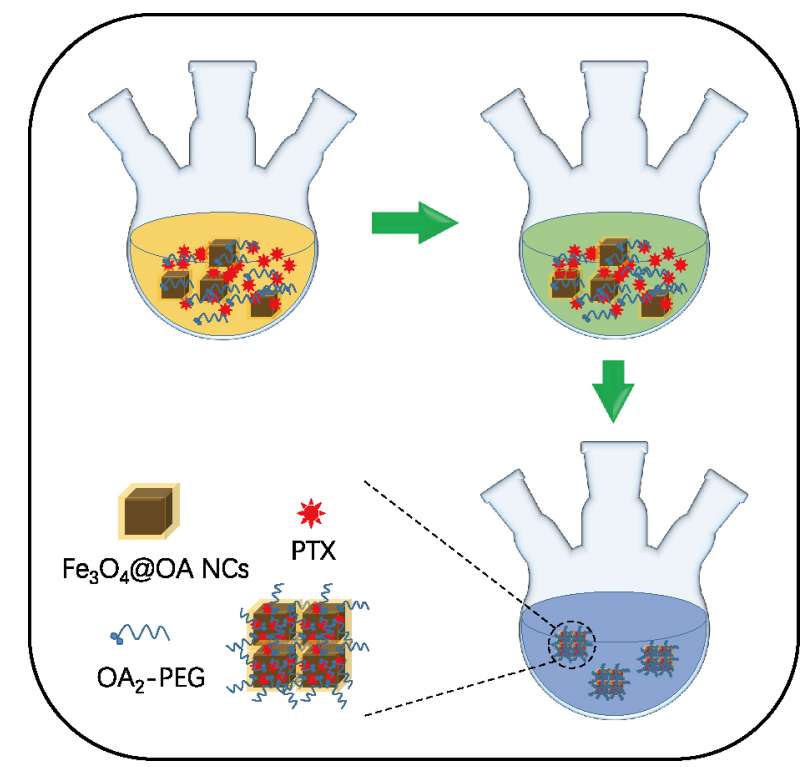

Figure 3 Schematic representation and structure of Rubik's cube-like PTX magnetic nanoassemblies, modified from reference [119], Copyright 2016, Elsevier.
MNPs and various crystal PLA domains on the surface of the PLA-nanoparticle composites affected the behavior of the PLA during hydrolysis.

\section{Magnetic microbubbles and liposomes as multimodality theranostic delivery systems}

Apart from the direct use of MNPs as a drug adsorption platform for in vivo delivery, many authors have reported microcapsule structures that encapsulate MNPs in organic or inorganic shells [126], where the microcapsules act as an effective platform for the simultaneous delivery of encapsulated drugs and MNPs. Drugs or other biologically active substances can be embedded in the internal area of the microcapsules, thereby preventing the drugs from contacting healthy tissue before reaching the disease site or the specific tissue. The shell structure of the microcapsules is then changed by the application of an external magnetic field and/or physiological microenvironment triggers. This triggers the release of the drug from the microcapsules. The structure could be called a "smart" magnetic nanodevice owing to the precision by which the drug release is achieved to optimal effect. Yang et al. [127] designed a micro-container embedded with $\mathrm{Fe}_{3} \mathrm{O}_{4}$ nanoparticles. Nitric oxide precursor drugs and 1 -arginine were also encapsulated in the core of the micro-container. Under the stimulation of an alternating magnetic field, the permeability of microcapsule membrane was changed, and $\mathrm{H}_{2} \mathrm{O}_{2}$, which commonly resides in the inflammatory region, is released into the interior resulting in a bubble microreactor formation in situ. The reaction, product nitrogen oxide (NO), is beneficial because it alleviates tissue inflammation and acts as an ultrasound contrast agent to monitor targeted tissue with ultrasound imaging. Thus, the magnetic field-triggered NO microbubbles can be utilized like a very simple, but important and effective magnetic nanodevice to achieve the diagnostic and therapeutic goal.

Subsequently, further experiments were designed to investigate the use of magnetic microbubbles with therapeutic capabilities. After binding to tumor-targeted biomolecules, the magnetic microbubbles can be developed as molecularly targeted imaging DDSs. In one experiment, arginine-glycine-aspartic acid-L-tumor necrosis factor-related apoptosis-inducing ligand (RGD-L-TRAIL), an antitumor-targeting fusion protein, was precisely conjugated to the surface of the MNP-coated microbubbles to construct RGD molecularly targeted magnetic microbubbles (RGD-L-TRAIL@MMBs) [128]. Owing to the highly specific accumulation of RGD-L-TRAIL@MMBs in the tumor, accurate diagnostic information about the tumor 
can be obtained by dual ultrasound and MRI (Fig. 4). After imaging, the TRAIL molecules act as anticancer agents and gain access to the interior of the cancer cells by nanoparticle- and RGD-mediated endocytosis to effectively induce tumor cell apoptosis. It is expected that a proper MNP-based microbubble DDS could be developed as a molecularly targeted multimodality imaging delivery system with the addition of chemotherapeutic cargoes to improve cancer diagnosis and therapy.

Finally, taking into account the superior biocompatibility of liposomes, magnetic liposomes are also frequently developed and applied. Liposomes are artificial phospholipid vesicles with a mean diameter of $50-1000 \mathrm{~nm}$. Both water-soluble and water-insoluble drugs/nanoparticles can be loaded into the inner core and hydrophobic bilayer, respectively, forming a promising DDS [129]. When the hydrophobic or hydrophilic MNPs are loaded into the liposome formulation, the fabricated magnetic liposome delivery systems can be remotely controlled via an external magnetic field. Traditionally, magnetic liposomes make excellent MR contrast agents. In particular, the MR of magnetic liposomes simultaneously facilitates image guidance of the liposomal drug delivery in the specific areas in vivo. An innovative class of magnetic hyperthermia and smart controllable liposomes have been developed. Sharifabad et al. [130] prepared liposome-capped core-shell mesoporous silica-coated SPIOs called "magnetic protocells" as novel nanocomposites, and used them for loading the anticancer

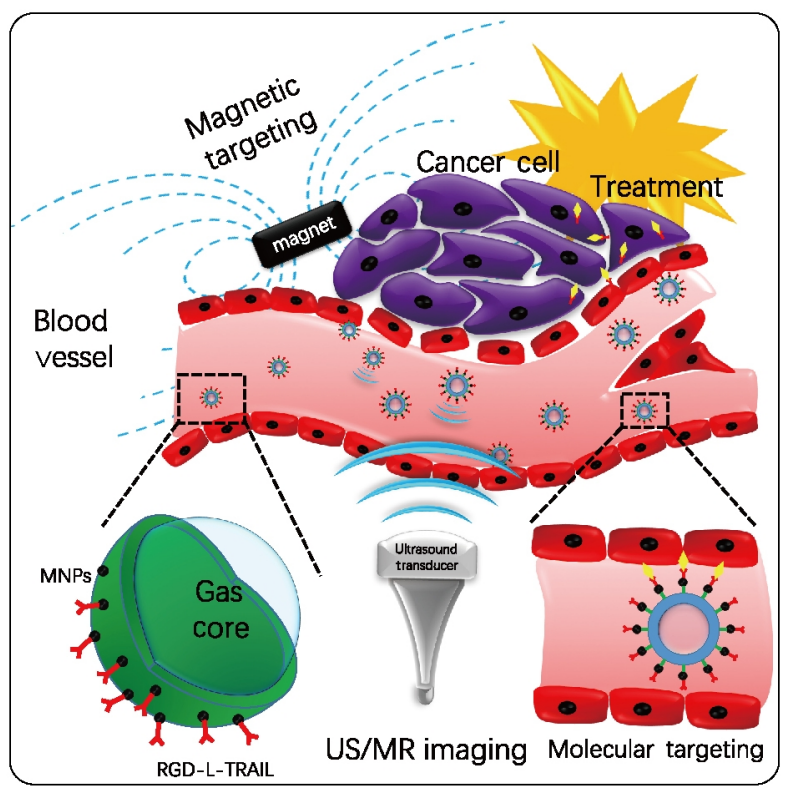

Figure 4 Schematic diagram showing the enhanced targeting strategy of RGD-L-TRAIL@MMBs for tumor diagnostics and therapy. drug DOX. The structure was adjusted to maintain a temperature of $43^{\circ} \mathrm{C}$ with a concentration of $10 \mathrm{mg} \mathrm{mL}^{-1}$ in a variable magnetic field with a maximum strength of 200 Gauss and a frequency of $406 \mathrm{kHz}$. A slow but linear increase in DOX release over time at $43^{\circ} \mathrm{C}$ was demonstrated to be favorable for drug delivery applications without affecting the structure of the capped liposomes. Liu et al. [131] designed a stimuli-responsive anethole dithiolethione (ADT)-loaded magnetic nanoliposome (AML) delivery system, which consists of ADT and a hydrogen sulfide $\left(\mathrm{H}_{2} \mathrm{~S}\right)$ pro-drug doped in the lipid bilayer, and superparamagnetic nanoparticles encapsulated inside. For in vivo applications, after preferentially targeting the tumor tissue when spatiotemporally navigated by an external magnetic field, the nanoscale AMLs can intratumorally convert to micro-sized $\mathrm{H}_{2} \mathrm{~S}$ bubbles. This dynamic process can be monitored by magnetic resonance and ultrasound dual modal imaging. Importantly, the intratumoral-generated $\mathrm{H}_{2} \mathrm{~S}$ bubbles visualized by real-time ultrasound imaging can first ablate the tumor tissue when exposed to higher acoustic intensity; then as gasotransmitters, intratumoral-generated high-concentration $\mathrm{H}_{2} \mathrm{~S}$ molecules can diffuse into the inner tumor regions where they have a further synergistic antitumor effect. The 7-day follow-up observations for tumor-bearing mice indicated that AMLs and magnetic field treatment greatly improved the inhibition of tumor growth.

Very recently, a cell membrane-mimicking lipid composition presented as a novel surface mask for MNPs has been shown to confer various biological benefits. It is reported that platelet (PLT) membrane-coated $\mathrm{Fe}_{3} \mathrm{O}_{4} \mathrm{MNPs}$, which may inherit prolonged blood circulation and cancer targeting capabilities from the PLT membranes, are a tentative design for personal therapy [71]. The $\mathrm{T}_{2}$-weighted relaxation rate value $\left(R_{2}\right)$ of the PLT-MNPs suggests that the surface membrane coating does not compromise MRI functionality. The in vivo MRI images confirmed that the PLT-MNPs have better tumor accumulation behavior than uncoated MNPs. Tumor photothermal therapy with PLTMNPs exhibited tumor temperature increases from 34.4 to $56.1^{\circ} \mathrm{C}$ within $5 \mathrm{~min}$. In contrast, the tumor temperatures of MNP and RBC-MNP groups in mice reached only 49.5 and $53.6^{\circ} \mathrm{C}$, respectively.

\section{CONCLUSIONS}

MNPs have emerged as excellent multifunctional nanoplatforms for the construction of smart DDSs. Numerous designs have been made in this field over the last 10 years, reflecting an exponentially growing number of 
publications. A search of the Web of Science that stipulated "magnetic nanoparticles" and "drug delivery systems" as two keywords produced 805 hits, and the total cited frequency was 21,184 hits. Moreover, $81.86 \%$ of the publications were original articles, $17.89 \%$ were reviews, and $4.48 \%$ were reports of proceedings, conference abstracts, and editorials. Furthermore, $27.70 \%$ of the publications were from the United States, $23.85 \%$ from China, and $6.83 \%$ from South Korea. The contributions from China are increasing sharply year on year.

With a gradual increase in clinical requirements, although most DDSs, including MNPs, are in mutually competitive development stages, MDDSs have become one of the most promising DDSs. MNPs with suitable physicochemical properties, modified with biocompatible polymeric, lipid, or metal shells, are applicable to a wide variety of biomedical fields, both diagnostic and therapeutic. As discussed, the size, charge, and surface features of MNPs could strongly influence their biodistribution, biotoxicity, and magnetism. Administrated MDDSs, utilizing external magnetic fields, can be transported to targeted diseased tissues, where the drugs can be released in a controllable manner. As a result of less drug cargo dosage and precise delivery, MDDSs do not exhibit serious side-effects. Even temperature increases arising from exposure to an alternating magnetic field have been investigated for tumor hyperthermia treatment. A number of positive in vivo results for MDDS suggest that work should progress from the laboratory to clinical trials.

However, before these MDDSs can be used commercially as diagnostic and therapeutic products, numerous challenges must be overcome. First, the in vivo metabolic processes in which these elaborate MDDSs are involved after injection are not very clear. To date, in vivo studies on the fate of iron are scarce, and macroscopic analysis of the quality distribution of MNPs in organs cannot explain the different mechanisms underlying specific cell phagocytosis, metabolism, degradation, and cell death. More emphasis should be put on detailed investigations of the in vivo properties of MNPs if these nanoparticles are to enter clinical trials. Continuing from this unsolved problem, more experimental data are needed for a comprehensive understanding of what happens to the MDDSs in the long term. The toxicity of the MNPs is complex and depends on their size, geometry, surface features, and magnetism. Thus, from a regulatory standpoint, the in vivo safety of MNPs needs to be evaluated more carefully. Furthermore, it is known that the functions of MDDSs and the property of external magnetic fields are inseparable.
However, owing to the restrictions imposed by physical conditions and the differences between patients with different diseases, external magnetic fields should be carefully modulated for effective treatment. Until now, few studies have explored the influence of magnetic fields on MDDSs. Despite the numerous challenges faced when exploring specific favorable clinical applications, the in vitro and in vivo experimental results are encouraging. Any efforts that improve the properties of MDDSs in vivo and reduce their clinical cost will accelerate the development of magnetic theranostic delivery systems in the future.

Received 31 March 2017; accepted 9 May 2017; published online 1 June 2017

1 Barick KC, Singh S, Bahadur D, et al. Carboxyl decorated $\mathrm{Fe}_{3} \mathrm{O}_{4}$ nanoparticles for MRI diagnosis and localized hyperthermia. J Colloid Interface Sci, 2014, 418: 120-125

2 Shanehsazzadeh S, Lahooti A, Hajipour MJ, et al. External magnetic fields affect the biological impacts of superparamagnetic iron nanoparticles. Colloid Surf B-Biointerfaces, 2015, 136: 1107-1112

3 Lima-Tenório MK, Gómez Pineda EA, Ahmad NM, et al. Magnetic nanoparticles: in vivo cancer diagnosis and therapy. Int J Pharm, 2015, 493: 313-327

4 Chen R, Ling D, Zhao L, et al. Parallel comparative studies on mouse toxicity of oxide nanoparticle- and gadolinium-based T1 MRI contrast agents. ACS Nano, 2015, 9: 12425-12435

5 de Smet M, Langereis S, van den Bosch S, et al. Temperature-sensitive liposomes for doxorubicin delivery under MRI guidance. J Control Release, 2010, 143: 120-127

6 Wu H, Liu G, Zhang S, et al. Biocompatibility, MR imaging and targeted drug delivery of a rattle-type magnetic mesoporous silica nanosphere system conjugated with PEG and cancer-cell-specific ligands. J Mater Chem, 2011, 21: 3037-3045

7 Somasundaram VH, Pillai R, Malarvizhi G, et al. Biodegradable radiofrequency responsive nanoparticles for augmented thermal ablation combined with triggered drug release in liver tumors. ACS Biomater Sci Eng, 2016, 2: 768-779

8 Veiseh O, Gunn JW, Zhang M. Design and fabrication of magnetic nanoparticles for targeted drug delivery and imaging. Adv Drug Deliver Rev, 2010, 62: 284-304

9 El-Boubbou K, Ali R, Bahhari HM, et al. Magnetic fluorescent nanoformulation for intracellular drug delivery to human breast cancer, primary tumors, and tumor biopsies: beyond targeting expectations. Bioconjugate Chem, 2016, 27: 1471-1483

10 Hayashi K, Ono K, Suzuki H, et al. High-frequency, magneticfield-responsive drug release from magnetic nanoparticle/organic hybrid based on hyperthermic effect. ACS Appl Mater Interfaces, 2010, 2: 1903-1911

11 Tietze R, Zaloga J, Unterweger H, et al. Magnetic nanoparticle-based drug delivery for cancer therapy. Biochem Biophys Res Commun, 2015, 468: 463-470

12 Jeon H, Kim J, Lee YM, et al. Poly-paclitaxel/cyclodextrin-SPION nano-assembly for magnetically guided drug delivery system. J Control Release, 2016, 231: 68-76

13 Che RL, Bear JC, McNaughter PD, et al. A SPION-eicosane protective coating for water soluble capsules: evidence for on-demand drug release triggered by magnetic hyperthermia. Sci Rep, 2016, 6: 20271 
14 Wahajuddin, Arora S. Superparamagnetic iron oxide nanoparticles: magnetic nanoplatforms as drug carriers. Int J Nanomed, 2012, 7: 3445

15 Muthiah M, Lee SJ, Moon M, et al. Surface tunable polymersomes loaded with magnetic contrast agent and drug for image guided cancer therapy. J Nanosci Nanotechnol, 2013, 13: 1626-1630

16 Jenkins SI, Weinberg D, Al-Shakli AF, et al. 'Stealth' nanoparticles evade neural immune cells but also evade major brain cell populations: implications for PEG-based neurotherapeutics. J Control Release, 2016, 224: 136-145

17 Luo S, Zhang Y, Cao J, et al. Arginine modified polymeric micelles as a novel drug delivery system with enhanced endocytosis efficiency. Colloid Surf B-Biointerfaces, 2016, 148: 181-192

18 Mashhadi MA, Ramazani A, Tabatabaei Rezaei SJ, et al. Design and construction of multifunctional hyperbranched polymers coated magnetite nanoparticles for both targeting magnetic resonance imaging and cancer therapy. J Colloid Interface Sci, 2017, 490: $64-73$

19 Zhu YJ, Chen F. pH-responsive drug-delivery systems. Chem Asian J, 2015, 10: 284-305

20 Rao Y, Chen W, Liang X, et al. Epirubicin-loaded superparamagnetic iron-oxide nanoparticles for transdermal delivery: cancer therapy by circumventing the skin barrier. Small, 2015, 11: 239-247

21 Nagesh PKB, Johnson NR, Boya VKN, et al. PSMA targeted docetaxel-loaded superparamagnetic iron oxide nanoparticles for prostate cancer. Colloid Surf B-Biointerfaces, 2016, 144: 8-20

22 Pradhan L, Thakur B, Srivastava R, et al. Assessing therapeutic potential of magnetic mesoporous nanoassemblies for chemo-resistant tumors. Theranostics, 2016, 6: 1557-1572

23 Wang J, Gong C, Wang Y, et al. Magnetic nanoparticles with a $\mathrm{pH}$-sheddable layer for antitumor drug delivery. Colloid Surf B-Biointerfaces, 2014, 118: 218-225

24 Yang HW, Huang CY, Lin CW, et al. Gadolinium-functionalized nanographene oxide for combined drug and microRNA delivery and magnetic resonance imaging. Biomaterials, 2014, 35: 6534-6542

25 Ding Q, Liu D, Guo D, et al. Shape-controlled fabrication of magnetite silver hybrid nanoparticles with high performance magnetic hyperthermia. Biomaterials, 2017, 124: 35-46

26 Petros RA, DeSimone JM. Strategies in the design of nanoparticles for therapeutic applications. Nat Rev Drug Discov, 2010, 9: 615-627

27 Zhai Y, Han L, Wang P, et al. Superparamagnetic plasmonic nanohybrids: shape-controlled synthesis, TEM-induced structure evolution, and efficient sunlight-driven inactivation of bacteria. ACS Nano, 2011, 5: 8562-8570

28 Parveen S, Misra R, Sahoo SK. Nanoparticles: a boon to drug delivery, therapeutics, diagnostics and imaging. Nanomed-Nanotechnol Biol Med, 2012, 8: 147-166

29 Bertrand $\mathrm{N}, \mathrm{Wu} J, \mathrm{Xu}$ X, et al. Cancer nanotechnology: the impact of passive and active targeting in the era of modern cancer biology. Adv Drug Deliver Rev, 2014, 66: 2-25

30 Gratton SEA, Ropp PA, Pohlhaus PD, et al. The effect of particle design on cellular internalization pathways. Proc Natl Acad Sci USA, 2008, 105: 11613-11618

31 Champion JA, Mitragotri S. Shape induced inhibition of phagocytosis of polymer particles. Pharm Res, 2009, 26: 244-249

32 Cheng D, Li X, Zhang G, et al. Morphological effect of oscillating magnetic nanoparticles in killing tumor cells. Nanoscale Res Lett, 2014, 9: 195

33 He Q, Zhang J, Chen F, et al. An anti-ROS/hepatic fibrosis drug de- livery system based on salvianolic acid B loaded mesoporous silica nanoparticles. Biomaterials, 2010, 31: 7785-7796

34 Di Bona KR, Xu Y, Ramirez PA, et al. Surface charge and dosage dependent potential developmental toxicity and biodistribution of iron oxide nanoparticles in pregnant CD-1 mice. Reproduct Toxicol, 2014, 50: 36-42

35 Hirsch V, Kinnear C, Moniatte M, et al. Surface charge of polymer coated SPIONs influences the serum protein adsorption, colloidal stability and subsequent cell interaction in vitro. Nanoscale, 2013, 5: $3723-3732$

36 Joly L, Ybert C, Trizac E, et al. Hydrodynamics within the electric double layer on slipping surfaces. Phys Rev Lett, 2004, 93: 257805

$37 \mathrm{Xu}$ R. Shear plane and hydrodynamic diameter of microspheres in suspension. Langmuir, 1998, 14: 2593-2597

38 Yang W, Lee J, Hong S, et al. Difference between toxicities of iron oxide magnetic nanoparticles with various surface-functional groups against human normal fibroblasts and fibrosarcoma cells. Materials, 2013, 6: 4689-4706

39 Sun Z, Yathindranath V, Worden M, et al. Characterization of cellular uptake and toxicity of aminosilane-coated iron oxide nanoparticles with different charges in central nervous system-relevant cell culture models. Int J Nanomed, 2013, 8: 961

40 Kolosnjaj-Tabi J, Wilhelm C, Clément O, et al. Cell labeling with magnetic nanoparticles: opportunity for magnetic cell imaging and cell manipulation. J Nanobiotechnol, 2013, 11: S7

41 Durán JDG, Arias JL, Gallardo V, et al. Magnetic colloids as drug vehicles. J Pharm Sci, 2008, 97: 2948-2983

42 Sjögren CE, Johansson C, Nævestad A, et al. Crystal size and properties of superparamagnetic iron oxide (SPIO) particles. Magn Resonance Imaging, 1997, 15: 55-67

43 Rahaman $\mathrm{H}$, Nath $\mathrm{A}$, Paul $\mathrm{R}$, et al. $\mathrm{Fe}_{3} \mathrm{O}_{4}-\mathrm{Mn}_{3} \mathrm{O}_{4}$ nanocomposites with moderate magnetism for in vitro cytotoxicity studies on macrophages. RSC Adv, 2016, 6: 83146-83153

44 Pouponneau P, Leroux JC, Soulez G, et al. Co-encapsulation of magnetic nanoparticles and doxorubicin into biodegradable microcarriers for deep tissue targeting by vascular MRI navigation. Biomaterials, 2011, 32: 3481-3486

45 Bear JC, Patrick PS, Casson A, et al. Magnetic hyperthermia controlled drug release in the GI tract: solving the problem of detection. Sci Rep, 2016, 6: 34271

46 Rose LC, Bear JC, Southern P, et al. On-demand, magnetic hyperthermia-triggered drug delivery: optimisation for the GI tract. J Mater Chem B, 2016, 4: 1704-1711

47 Shan J, Wang L, Yu H, et al. Recent progress in $\mathrm{Fe}_{3} \mathrm{O}_{4}$ based magnetic nanoparticles: from synthesis to application. Mater Sci Tech, 2015, 27: 1-13

48 Viñas SL, Simeonidis K, Li ZA, et al. Tuning the magnetism of ferrite nanoparticles. J Magn Magn Mater, 2016, 415: 20-23

49 Valois CRA, Braz JM, Nunes ES, et al. The effect of DMSA-functionalized magnetic nanoparticles on transendothelial migration of monocytes in the murine lung via a $\beta 2$ integrin-dependent pathway. Biomaterials, 2010, 31: 366-374

50 Nishimura I, Oshima A, Shibuya K, et al. Acute and subchronic toxicity of $20 \mathrm{kHz}$ and $60 \mathrm{kHz}$ magnetic fields in rats. J Appl Toxicol, 2016, 36: 199-210

51 Ruiz A, Mancebo A, Beola L, et al. Dose-response bioconversion and toxicity analysis of magnetite nanoparticles. IEEE Magn Lett, 2016, 7: 1-5

52 Häfeli UO, Riffle JS, Harris-Shekhawat L, et al. Cell uptake and in vitro toxicity of magnetic nanoparticles suitable for drug delivery. Mol Pharm, 2009, 6: 1417-1428

53 Asín L, Goya GF, Tres A, et al. Induced cell toxicity originates den- 
dritic cell death following magnetic hyperthermia treatment. Cell Death Dis, 2013, 4: e596

54 Kim JE, Shin JY, Cho MH. Magnetic nanoparticles: an update of application for drug delivery and possible toxic effects. Arch Toxicol, 2012, 86: 685-700

55 Park EJ, Umh HN, Choi DH, et al. Magnetite- and maghemite-induced different toxicity in murine alveolar macrophage cells. Arch Toxicol, 2014, 88: 1607-1618

56 Pickard MR, Chari DM. Robust uptake of magnetic nanoparticles (MNPs) by central nervous system (CNS) microglia: implications for particle uptake in mixed neural cell populations. IJMS, 2010, 11: 967-981

57 Easo SL, Mohanan PV. Dextran stabilized iron oxide nanoparticles: synthesis, characterization and in vitro studies. Carbohydrate Polym, 2013, 92: 726-732

58 Bautista MC, Bomati-Miguel O, del Puerto Morales M, et al. Surface characterisation of dextran-coated iron oxide nanoparticles prepared by laser pyrolysis and coprecipitation. J Magn Magn Mater, 2005, 293: 20-27

59 Khalkhali M, Sadighian S, Rostamizadeh K, et al. Synthesis and characterization of dextran coated magnetite nanoparticles for diagnostics and therapy. Bioimpacts, 2015, 5: 141-150

60 Remya NS, Syama S, Sabareeswaran A, et al. Toxicity, toxicokinetics and biodistribution of dextran stabilized iron oxide nanoparticles for biomedical applications. Int J Pharm, 2016, 511: 586-598

61 Peeples B, Goornavar V, Peeples C, et al. Structural, stability, magnetic, and toxicity studies of nanocrystalline iron oxide and cobalt ferrites for biomedical applications. J Nanopart Res, 2014, 16: 2290

62 Mejías R, Gutiérrez L, Salas G, et al. Long term biotransformation and toxicity of dimercaptosuccinic acid-coated magnetic nanoparticles support their use in biomedical applications. J Control Release, 2013, 171: 225-233

63 Bae JE, Huh MI, Ryu BK, et al. The effect of static magnetic fields on the aggregation and cytotoxicity of magnetic nanoparticles. Biomaterials, 2011, 32: 9401-9414

64 Luo Y, Zhou Z, Yue T. Synthesis and characterization of nontoxic chitosan-coated $\mathrm{Fe}_{3} \mathrm{O}_{4}$ particles for patulin adsorption in a juice- $\mathrm{pH}$ simulation aqueous. Food Chem, 2017, 221: 317-323

65 Barbosa-Barros L, García-Jimeno S, Estelrich J. Formation and characterization of biobased magnetic nanoparticles double coated with dextran and chitosan by layer-by-layer deposition. Colloid Surf A-Physicochem Eng Aspects, 2014, 450: 121-129

66 Wang Y, Xu F, Zhang L, et al. One-pot solvothermal synthesis of $\mathrm{Fe}_{3} \mathrm{O}_{4}$-PEI composite and its further modification with $\mathrm{Au}$ nanoparticles. J Nanopart Res, 2013, 15: 1338

67 Xiong L, Jiang HW, Wang DZ. Preparation and characterization of PVP- $b$-PLA modified $\mathrm{Fe}_{3} \mathrm{O}_{4}$ magnetic nanoparticles. Acta Polym Sinica, 2008, 8: 791-796

68 Lee SY, Ahn CY, Lee J, et al. Rapid and selective separation for mixed proteins with thiol functionalized magnetic nanoparticles. Nanoscale Res Lett, 2012, 7: 279

69 Chen JP, Yang PC, Ma YH, et al. Superparamagnetic iron oxide nanoparticles for delivery of tissue plasminogen activator. J Nanosci Nanotechnol, 2011, 11: 11089-11094

70 Singh RK, Kim TH, Patel KD, et al. Biocompatible magnetite nanoparticles with varying silica-coating layer for use in biomedicine: physicochemical and magnetic properties, and cellular compatibility. J Biomed Mater Res, 2012, 100A: 1734-1742

71 Rao L, Bu LL, Meng QF, et al. Antitumor platelet-mimicking magnetic nanoparticles. Adv Funct Mater, 2017, 27: 1604774

72 Shi F, Hui W, Cui Y, et al. surface modification and characterization of $\mathrm{Fe}_{3} \mathrm{O}_{4} / \mathrm{Au}$ composite nanoparticles. Nano, 2011, 06: 145-151
73 Kalska-Szostko B, Hilgendorff M, Giersig M, et al. Magneto-optical properties of Co nanoparticles/(Cu, $\mathrm{Ag}, \mathrm{Au})$ sandwich systems. sci adv mater, 2015, 7: 540-545

74 Fuertges F, Abuchowski A. The clinical efficacy of poly(ethylene glycol)-modified proteins. J Control Release, 1990, 11: 139-148

75 Yuan G, Yuan Y, Xu K, et al. Biocompatible PEGylated $\mathrm{Fe}_{3} \mathrm{O}_{4}$ nanoparticles as photothermal agents for near-infrared light modulated cancer therapy. IJMS, 2014, 15: 18776-18788

76 Illés E, Tombácz E, Szekeres M, et al. Novel carboxylated PEG-coating on magnetite nanoparticles designed for biomedical applications. J Magn Magn Mater, 2015, 380: 132-139

77 Kumagai M, Sarma TK, Cabral H, et al. Enhanced in vivo magnetic resonance imaging of tumors by PEGylated iron-oxide-gold core-shell nanoparticles with prolonged blood circulation properties. Macromol Rapid Commun, 2010, 31: 1521-1528

78 Khandhar AP, Keselman P, Kemp SJ, et al. Evaluation of PEGcoated iron oxide nanoparticles as blood pool tracers for preclinical magnetic particle imaging. Nanoscale, 2017, 9: 1299-1306

79 Larsen EKU, Nielsen T, Wittenborn T, et al. Size-dependent accumulation of PEGylated silane-coated magnetic iron oxide nanoparticles in murine tumors. ACS Nano, 2009, 3: 1947-1951

80 Dung DTK, Hai TH, Phuc LH, et al. Preparation and characterization of magnetic nanoparticles with chitosan coating. J Phys-Conf Ser, 2009, 187: 012036

81 López RG, Pineda MG, Hurtado G, et al. Chitosan-coated magnetic nanoparticles prepared in one step by reverse microemulsion precipitation. IJMS, 2013, 14: 19636-19650

82 Ahmad T, Bae H, Iqbal Y, et al. Chitosan-coated nickel-ferrite nanoparticles as contrast agents in magnetic resonance imaging. J Magn Magn Mater, 2015, 381: 151-157

83 Dorniani D, Hussein MZB, Kura AU, et al. Sustained release of prindopril erbumine from its chitosan-coated magnetic nanoparticles for biomedical applications. IJMS, 2013, 14: 23639-23653

84 Zhao F, Zhang B, Wang J, et al. Synthesis and properties of magnetite nanoparticles coated with poly(ethylene glycol) and poly(ethylene imine). J Nanosci Nanotechnol, 2013, 13: 6793-6797

85 Schweiger C, Pietzonka C, Heverhagen J, et al. Novel magnetic iron oxide nanoparticles coated with poly(ethylene imine)-g-poly(ethylene glycol) for potential biomedical application: synthesis, stability, cytotoxicity and MR imaging. Int J Pharm, 2011, 408: 130-137

86 Hong RY, Li JH, Qu JM, et al. Preparation and characterization of magnetite/dextran nanocomposite used as a precursor of magnetic fluid. Chem Eng J, 2009, 150: 572-580

87 Liu G, Hong RY, Guo L, et al. Preparation, characterization and MRI application of carboxymethyl dextran coated magnetic nanoparticles. Appl Surface Sci, 2011, 257: 6711-6717

88 Wagner K, Kautz A, Röder M, et al. Synthesis of oligonucleotidefunctionalized magnetic nanoparticles and study on their in vitro cell uptake. Appl Organometal Chem, 2004, 18: 514-519

89 Li X, Peng M, Raju PA, et al. Dextran-coated goldmag nanoparticles enhance the colloidal stability and controlled-release of doxorubicin. IEEE Trans Magn, 2013, 49: 359-363

90 Salunkhe AB, Khot VM, Thorat ND, et al. Polyvinyl alcohol functionalized cobalt ferrite nanoparticles for biomedical applications. Appl Surface Sci, 2013, 264: 598-604

91 Winnik F, Qiu X. Preparation and characterization of PVA coated magnetic nanoparticles. Chin J Polym Sci, 2000, 18: 535-539

92 Hajikarimi Z, Khoei S, Khoee S, et al. Evaluation of the cytotoxic effects of PLGA coated iron oxide nanoparticles as a carrier of 5-fluorouracil and mega-voltage X-ray radiation in DU145 prostate cancer cell line. IEEE Trans Nanobiosci, 2014, 13: 403-408

93 Jaberolansar E, Kameli P, Ahmadvand H, et al. Synthesis and char- 
acterization of PVP-coated $\mathrm{Co}_{0.3} \mathrm{Zn}_{0.7} \mathrm{Fe}_{2} \mathrm{O}_{4}$ ferrite nanoparticles. J Magn Magn Mater, 2016, 404: 21-28

94 Karimzadeh I, Aghazadeh M, Ganjali MR, et al. A novel method for preparation of bare and poly(vinylpyrrolidone) coated superparamagnetic iron oxide nanoparticles for biomedical applications. Mater Lett, 2016, 179: 5-8

95 Rose PA, Praseetha PK, Bhagat M, et al. Drug embedded PVP coated magnetic nanoparticles for targeted killing of breast cancer cells. Technol Cancer Res Treat, 2013, 12: 463-472

96 Huang J, Bu L, Xie J, et al. Effects of nanoparticle size on cellular uptake and liver MRI with polyvinylpyrrolidone-coated iron oxide nanoparticles. ACS Nano, 2010, 4: 7151-7160

97 Wang W, Jing Y, He S, et al. Surface modification and bioconjugation of FeCo magnetic nanoparticles with proteins. Colloid Surf B-Biointerfaces, 2014, 117: 449-456

98 Yang HW, Hua MY, Lin KJ, et al. Bioconjugation of recombinant tissue plasminogen activator to magnetic nanocarriers for targeted thrombolysis. Int J Nanomed, 2012, 7: 5159

99 Rao L, Xu JH, Cai B, et al. Synthetic nanoparticles camouflaged with biomimetic erythrocyte membranes for reduced reticuloendothelial system uptake. Nanotechnology, 2016, 27: 085106

100 Liu Y. Research development on the preparation of gold-coated magnetic nanoparticles. AMR, 2011, 391-392: 381-384

101 Sun TW, Zhu YJ, Chen F, et al. Superparamagnetic yolk-shell porous nanospheres of iron oxide@magnesium silicate: synthesis and application in high-performance anticancer drug delivery. RSC Adv, 2016, 6: 103399-103411

102 Iqbal Y, Bae H, Rhee I, et al. Magnetic heating of silica-coated manganese ferrite nanoparticles. J Magn Magn Mater, 2016, 409: 80-86

103 Iqbal Y, Bae H, Rhee I, et al. Intensive analysis of core-shell silica-coated iron-oxide nanoparticles for magnetic hyperthermia. J Nanosci Nanotechnol, 2016, 16: 11862-11867

104 Li C, Ma C, Wang F, et al. Preparation and biomedical applications of core-shell silica/magnetic nanoparticle composites. J Nanosci Nanotechnol, 2012, 12: 2964-2972

105 Rho WY, Kim HM, Kyeong S, et al. Facile synthesis of monodispersed silica-coated magnetic nanoparticles. J Industrial Eng Chem, 2014, 20: 2646-2649

106 Shen X, Ge Z, Pang Y. Conjugating folate on superparamagnetic $\mathrm{Fe}_{3} \mathrm{O}_{4} @ \mathrm{Au}$ nanoparticles using click chemistry. J Solid State Chem, 2015, 222: 37-43

107 Poudyal N, Gandha KH, Liu J, et al. Ferromagnetic FePt/Au core/shell nanoparticles prepared by solvothermal annealing. IEEE Magn Lett, 2016, 7: 1-5

108 Wang J, Wu X, Wang C, et al. Facile synthesis of Au-coated magnetic nanoparticles and their application in bacteria detection via a sers method. ACS Appl Mater Interfaces, 2016, 8: 19958-19967

109 Chen Y, Gao N, Jiang J. Surface matters: enhanced bactericida property of core-shell Ag- $\mathrm{Fe}_{2} \mathrm{O}_{3}$ nanostructures to their heteromer counterparts from one-pot synthesis. Small, 2013, 9: 3242-3246

110 Bian L, Bao L, Wang J, et al. In situ preparation of monodispersed $\mathrm{Ag} /$ polyaniline $/ \mathrm{Fe}_{3} \mathrm{O}_{4}$ nanoparticles via heterogeneous nucleation. Nanoscale Res Lett, 2013, 8: 309

111 Chen Z, Yin JJ, Zhou YT, et al. Dual enzyme-like activities of iron oxide nanoparticles and their implication for diminishing cytotoxicity. ACS Nano, 2012, 6: 4001-4012

112 Yang F, Li M, Cui H, et al. Altering the response of intracellular reactive oxygen to magnetic nanoparticles using ultrasound and microbubbles. Sci China Mater, 2015, 58: 467-480

113 Xiong F, Wang H, Feng Y, et al. Cardioprotective activity of iron oxide nanoparticles. Sci Rep, 2015, 5: 8579

114 Zhao P, Cao M, Song L, et al. Downregulation of MIM protein inhibits the cellular endocytosis process of magnetic nanoparticles in macrophages. RSC Adv, 2016, 6: 96635-96643

115 Ford GC, Harrison PM, Rice DW, et al. Ferritin: design and formation of an iron-storage molecule. Philos Trans R Soc B-Biol Sci, 1984, 304: 551-565

116 Zhang W, Zhang Y, Chen Y, et al. Prussian blue modified ferritin as peroxidase mimetics and its applications in biological detection. J Nanosci Nanotechnol, 2013, 13: 60-67

117 Zhang W, Hu S, Yin JJ, et al. Prussian blue nanoparticles as multienzyme mimetics and reactive oxygen species scavengers. J Am Chem Soc, 2016, 138: 5860-5865

118 Yang $\mathrm{F}, \mathrm{Hu} \mathrm{S}$, Zhang Y, et al. A hydrogen peroxide-responsive $\mathrm{O}_{2}$ nanogenerator for ultrasound and magnetic-resonance dual modality imaging. Adv Mater, 2012, 24: 5205-5211

119 Wang Q, Chen B, Cao M, et al. Response of MAPK pathway to iron oxide nanoparticles in vitro treatment promotes osteogenic differentiation of hBMSCs. Biomaterials, 2016, 86: 11-20

120 Liu X, Zhang J, Tang S, et al. Growth enhancing effect of LBLassembled magnetic nanoparticles on primary bone marrow cells. Sci China Mater, 2016, 59: 901-910

121 Wang Q, Chen B, Ma F, et al. Magnetic iron oxide nanoparticles accelerate osteogenic differentiation of mesenchymal stem cells via modulation of long noncoding RNA INZEB2. Nano Res, 2017, 10: 626-642

122 Liu D, Wu W, Chen X, et al. Conjugation of paclitaxel to iron oxide nanoparticles for tumor imaging and therapy. Nanoscale, 2012, 4: 2306-2310

123 Xiong F, Chen Y, Chen J, et al. Rubik-like magnetic nanoassemblies as an efficient drug multifunctional carrier for cancer theranostics. J Control Release, 2013, 172: 993-1001

124 Panyam J, Labhasetwar V. Biodegradable nanoparticles for drug and gene delivery to cells and tissue. Adv Drug Deliver Rev, 2003, 55: 329-347

125 Yang F, Zhang X, Song L, et al. Controlled drug release and hydrolysis mechanism of polymer-magnetic nanoparticle composite. ACS Appl Mater Interfaces, 2015, 7: 9410-9419

126 Lu BQ, Zhu YJ, Chen F, et al. Core-shell hollow microspheres of magnetic iron oxide@amorphous calcium phosphate: synthesis using adenosine 5 '-triphosphate and application in $\mathrm{pH}$-responsive drug delivery. Chem Asian J, 2014, 9: 2908-2914

127 Yang F, Chen $\mathrm{P}, \mathrm{He} \mathrm{W}$, et al. Bubble microreactors triggered by an alternating magnetic field as diagnostic and therapeutic delivery devices. Small, 2010, 6: 1300-1305

128 Duan L, Yang F, He W, et al. A multi-gradient targeting drug delivery system based on RGD-L-TRAIL-labeled magnetic microbubbles for cancer theranostics. Adv Funct Mater, 2016, 26: 8313-8324

129 Torchilin VP. Fluorescence microscopy to follow the targeting of liposomes and micelles to cells and their intracellular fate. Adv Drug Deliver Rev, 2005, 57: 95-109

130 Sharifabad ME, Mercer T, Sen T. Drug-loaded liposome-capped mesoporous core-shell magnetic nanoparticles for cellular toxicity study. Nanomedicine, 2017, 11: 2757-2767

131 Liu Y, Yang F, Yuan C, et al. Magnetic nanoliposomes as in situ microbubble bombers for multimodality image-guided cancer theranostics. ACS Nano, 2017, 11: 1509-1519

Acknowledgments This work was financially funded by the National Natural Science Foundation of China (NSFC, 31370019, 61420106012) and the project of National Key Basic Research Program of China (2013CB733804). The funding partially comes from the Fundamental Research Funds for the Central Universities (2242016K41072), Zhong Ying Young Scholar of Southeast University as well as the support from 
the Collaborative Innovation Center of Suzhou Nano Science and Technology.

Author contributions $\mathrm{Gu} \mathrm{N}$ and Yang F provided the overall concept. Liu Y wrote the paper and Li M prepared the figures, table and co-wrote the section of "SURFACE FUNCTIONALIZATION OF MAGNETIC NANOPARTICLES FOR TARGETING IN VIVO". Yang F revised the manuscript. All authors participated in the discussion about the overall manuscript.
Conflict of interest The authors declare that they have no conflict of interest.

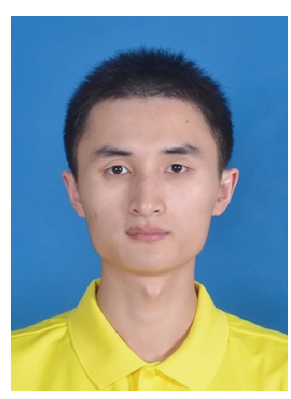

Yang Liu received his BSc degree from Southeast University, Nanjing, in 2015. Now, he is a PhD student in Prof. Fang Yang's group in Southeast University. His research interests are focused on the ultrasound imaging guided drug delivery system and designing novel multi-model sensitive nanoparticles.
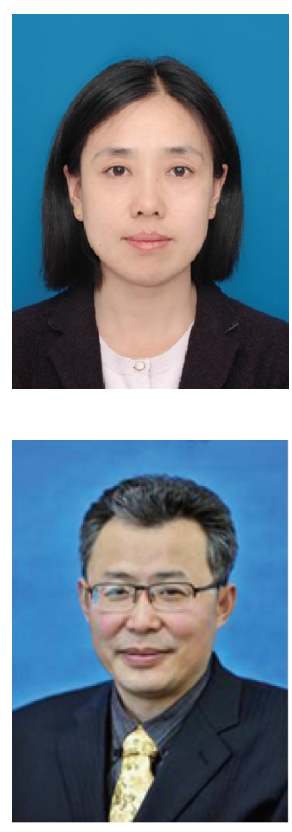

Fang Yang was born in 1979. She received her PhD degree in biomedical engineering from the School of Biological Science and Medical Engineering, Southeast University in 2009. Currently, she is a professor of the School of Biological Science and Medical Engineering, Southeast University. Her research interests mainly focus on the ultrasound multi-modal imaging, magnetic microbubbles, ultrasound molecular imaging, and imaging (ultrasound, magnetic resonance, optical, CT, etc.) guided accurate drug delivery system, etc.
Ning Gu was born in 1964. He received his PhD degree in biomedical engineering from the Department of Biomedical Engineering, Southeast University, Nanjing, China, in 1996. Currently he is a Changjiang Scholar Professor and NSFC Outstanding Young Investigator Fund Winner at Southeast University. He also serves as the president of Jiangsu Society of Biomedical Engineering, the director of the Research Center for Nanoscale Science and Technology of Southeast University, and the chief researcher of the Collaborative Innovation Center of Suzhou Nano-Science and Technology (2011 Program). His research interests include magnetic nanobiomaterials, nanobiology, medical imaging, advanced instrument development, etc.

\section{基于磁性质的药物递送系统}

刘洋, 李明喜, 杨芳*, 顾宁*

摘要 随着过去几十年来生物医学纳米技术和纳米材料领域的持续发展, 基于纳米颗粒的药物输送系统逐渐开始有望应用于临床研究. 其 中, 由于具有良好的生物相容性和优异的多功能负载能力, 基于磁性纳米粒子的磁性药物传递系统受到越来越多的关注. 本综述首先总结 了磁性药物传递系统的基本物理化学性质, 以阐明磁性药物传递系统需要保持适当的性能以满足特定的临床需要; 其次, 讨论了在设计未 来临床应用的磁性药物传递系统时的表面修饰和功能化问题; 最后, 重点综述了磁性纳米颗粒、磁性组装体以及磁性微泡、磁性脂质体和 生物膜修饰的磁性载体系统的设计和制备最新进展. 最后, 本综述对目前研究的磁性载体系统的设计、制备和安全性进行了总结, 并对未 来进一步解决磁性药物传递系统的临床应用瓶颈和前景进行了展望. 